\title{
Finding Suitable Clinical Endpoints for a Potential Treatment of a Rare Genetic Disease: the Case of ARID1B
}

\author{
Matthijs D. Kruizinga ${ }^{1,2}$ (D) $\cdot$ Rob G.J.A. Zuiker $^{1} \cdot$ Elif Sali $^{1} \cdot$ Marieke L. de Kam $^{1} \cdot$ Robert J. Doll $^{1} \cdot$ Geert Jan Groeneveld $^{1}$. \\ Gijs W.E. Santen ${ }^{3} \cdot$ Adam F. Cohen $^{1}$
}

Published online: 22 May 2020

(C) The Author(s) 2020

\begin{abstract}
There is a lack of reliable, repeatable, and non-invasive clinical endpoints when investigating treatments for intellectual disability (ID). The aim of this study is to explore a novel approach towards developing new endpoints for neurodevelopmental disorders, in this case for ARID1B-related ID. In this study, twelve subjects with ARID1B-related ID and twelve age-matched controls were included in this observational case-control study. Subjects performed a battery of non-invasive neurobehavioral and neurophysiological assessments on two study days. Test domains included cognition, executive functioning, and eye tracking. Furthermore, several electrophysiological assessments were performed. Subjects wore a smartwatch (Withings ${ }^{\circledR}$ Steel HR) for 6 days. Tests were systematically assessed regarding tolerability, variability, repeatability, difference with control group, and correlation with traditional endpoints. Animal fluency, adaptive tracking, body sway, and smooth pursuit eye movements were assessed as fit-forpurpose regarding all criteria, while physical activity, heart rate, and sleep parameters show promise as well. The event-related potential waveform of the passive oddball and visual evoked potential tasks showed discriminatory ability, but EEG assessments were perceived as extremely burdensome. This approach successfully identified fit-for-purpose candidate endpoints for ARID1B-related ID and possibly for other neurodevelopmental disorders. Next, results could be replicated in different ID populations or the assessments could be included as exploratory endpoint in interventional trials in ARID1B-related ID.
\end{abstract}

Key Words ARID1B $\cdot$ Endpoint $\cdot$ Biomarkers $\cdot$ Electrophysiology $\cdot$ Executive functioning $\cdot$ Eye tracking $\cdot$ Cognition

\begin{abstract}
Abbreviations
ABC Aberrant behavior checklist

ASSR Auditory steady-state response

ARID1B AT-rich interaction domain 1B

CHDR Centre for Human Drug Research

The authors confirm that the PI for this paper is Rob GJA Zuiker and that he had direct clinical responsibility for patients and volunteers.

Electronic supplementary material The online version of this article (https://doi.org/10.1007/s13311-020-00868-9) contains supplementary material, which is available to authorized users.
\end{abstract}

Matthijs D. Kruizinga

mkruizinga@chdr.nl

1 Centre for Human Drug Research, Zernikedreef 8, 2333 CL Leiden, the Netherlands

2 Juliana Children's Hospital, HAGA Teaching Hospital, the Hague, the Netherlands

3 Department of Clinical Genetics, Leiden University Medical Centre, Leiden, the Netherlands

$\begin{array}{ll}\text { CNS } & \text { Central nervous system } \\ \text { CV } & \text { Coefficient of variability } \\ \text { EEG } & \text { Electroencephalography } \\ \text { ERP } & \text { Event-related potentials } \\ \text { ID } & \text { Intellectual disability } \\ \text { ITPC } & \text { Inter-trial phase coherence } \\ \text { IQ } & \text { Intelligence quotient } \\ \text { MDES } & \text { Minimal detectable effect size } \\ \text { VEP } & \text { Visual evoked potentials } \\ \text { VVLT } & \text { Visual verbal learning test }\end{array}$

\section{Introduction}

Historically, treatment of intellectual disability (ID) and other neurodevelopmental disorders has focused primarily on the symptoms. Except for a few enzyme deficiency disorders, no treatments of underlying etiology have been incorporated in standard care [1]. Although animal models mimicking clinical ID syndromes have shown promising preclinical data, 
subsequent trials in humans have failed to show beneficial treatment effects $[2,3]$. While translation from mice to humans with ID seems unpredictable, it is generally accepted that a lack of reliable clinical endpoints plays a large role in this disparity. [4] Before conducting further interventional trials in subjects and children with ID, new trial designs and especially endpoints are needed [5]. In this study, we investigate the syndrome ARID1B-related ID as a model to illustrate how to develop new biomarkers for a rare neurodevelopmental disorder.

ARID1B-related ID is caused by haploinsufficiency of $A R I D 1 B$. Pathogenic variants in ARIDIB have been identified as a cause of Coffin-Siris syndrome in 2012 for the first time $[6,7]$. Since then, over 143 patients have been identified and the gene has now been associated with a variable array of phenotypes, ranging from Coffin-Siris syndrome to mild behavioral abnormalities [8]. Most commonly, patients suffer from ID, speech and vision impairment, and (partial) agenesis of the corpus callosum, and display distinct facial features [8, 9]. Mice with Arid1b haploinsufficiency showed similar symptoms and were found to have a reduced number of inhibitory GABA-ergic interneurons, causing a presumed inhibition-excitation imbalance which could be partly reversed by the GABA-A positive allosteric modulator clonazepam [10]. Since clonazepam is considered safe, it is a good candidate drug to investigate in patients. However, without fitfor-purpose endpoints, a trial would be doomed to fail.

Central nervous system (CNS) endpoints in ID trials should be considered fit-for-purpose when they satisfy a number of criteria [11]. In our opinion, they must reflect neurological and functional aspects relevant to the disease and be sensitive to detect pharmacological CNS effects. In the case of ID, endpoints should also be non-invasive and easily conducted. Repeatability should be determined in the targeted population and there should be a clear differentiation between patients and control subjects. Ideally, test results should correlate with existing indicators of disease severity.

The NeuroCart巴 is a neurological test battery known to be sensitive for the detection of CNS effects of compounds [12]. Using this test battery, non-invasive and data-intensive studies can be performed to demonstrate specific, time- and dosedependent, neurophysiological, and neuropsychological effects [12]. However, the assessments have not been investigated in patients with ID yet. The aim of this study is to explore the characteristics of a battery of non-invasive neurophysiological and neurobehavioral assessments that may be fit-for-purpose as future clinical endpoints for ARID1Brelated ID and similar syndromes.

\section{Materials and Methods}

This study was conducted at the Centre of Human Drug Research (CHDR) in Leiden, the Netherlands, from
November 2018 until May 2019 and the protocol was reviewed and approved by the Beoordeling Ethiek Biomedisch Onderzoek (BEBO) Foundation Review Board (Assen, the Netherlands). The study was conducted according to the Dutch Act on Medical Research Involving Human Subjects, the Dutch codes of conduct regarding medical research with minors and expression of objection by people with mental disabilities and in compliance with good clinical practice.

\section{Subjects and Study Design}

During this case-control study, twelve patients with pathogenic variants in $A R I D I B$ were recruited via the Coffin-Siris expertise center of the Leiden University Medical Centre. Twelve age-matched healthy controls were also recruited. Age difference between patients and controls was no more than 2 years, except for adult subjects. Subjects who regularly used benzodiazepines were excluded from the study. Tests were assessed for suitability for two age groups (2-4 and $\geq$ 5 years old), based on the expected capabilities of the subjects. Tests were performed on two consecutive Saturdays and were repeated 2-4 times during the study. The schedule of assessments is listed in Supplementary Figure S1. Study visits lasted 3-5 h. Baseline characteristics, including the last measured intelligence quotient (IQ) score, were obtained from patient charts. Parents completed the Aberrant Behavior Checklist $(\mathrm{ABC})$ at the start of the study [13].

\section{Selection of Candidate Endpoints}

NeuroCart® tests were selected based on the following criteria: (1) Tests must have demonstrated potential in detecting CNS effects of compounds; (2) Tests must investigate a CNS domain assumed to be affected in patients with ARID1B-related ID; (3) It must be reasonably expected that the tests can be conducted by children and patients with ARID1B-related ID; (4) Ideally, an improvement in test outcomes potentially results in symptom reduction or improvement of quality of life. Selected tests and the accompanying rationale for inclusion are listed in Table 1.

\section{Test Procedures}

\section{Cognition}

For the animal fluency test, subjects were asked to verbally produce as many different animals as they could sum up within $60 \mathrm{~s}$ [14]. Animals that were named twice or more did not count towards the total amount of animals named and neither were infant versions of adult animals already named. During the visual verbal learning test (VVLT), subjects were presented 30 words in three consecutive word trials [15]. Each trial ended with a free recall of the presented words. Thirty minutes 
Table 1 Rationale for selected tests

\begin{tabular}{|c|c|c|c|}
\hline & Test & CNS domain & Corresponding ARID1B symptom \\
\hline \multirow[t]{3}{*}{ Cognition } & Animal fluency test & Verbal fluency, semantic memory & Intellectual disability \\
\hline & VVLT & Memory & Intellectual disability \\
\hline & Day-night test & Memory and controlled processing & Impulsiveness and intellectual disability \\
\hline Eye tracking & $\begin{array}{l}\text { Smooth pursuit } \\
\text { Saccadic eye movements }\end{array}$ & $\begin{array}{l}\text { Attention and oculomotor function } \\
\text { Sedation }\end{array}$ & $\begin{array}{l}\text { Expected marker for clonazepam effect } \\
\text { Expected marker for clonazepam effect }\end{array}$ \\
\hline \multirow[t]{3}{*}{ Executive functioning } & Adaptive tracking & Motor activation and attention & Short attention span \\
\hline & Finger tapping & Motor activation and fluency & Lethargy and slowness \\
\hline & Body sway & Balance and attention & Hyperactivity \\
\hline \multirow[t]{4}{*}{ Electrophysiology } & $\begin{array}{l}\text { Resting EEG } \\
\text { Passive oddball }\end{array}$ & $\begin{array}{l}\text { General CNS activity } \\
\text { Auditory processing }\end{array}$ & $\begin{array}{l}\text { Hypothesized abnormal neuronal } \\
\text { organization and general CNS functioning }\end{array}$ \\
\hline & Active oddball & Auditory processing & Hypothesized abnormal neuronal \\
\hline & VEP & Visual processing & organization and general CNS functioning \\
\hline & ASSR & Auditory processing & \\
\hline \multirow[t]{3}{*}{ trial@home } & Steel HR_physical activity & General daily activity & Hyperactivity, apathy, and lethargy \\
\hline & Steel HR_-sleep parameters & Sleep & Insomnia \\
\hline & Steel HR — heart rate & Sympathetic activation and arousal & Hyperactivity \\
\hline
\end{tabular}

VVLT = visual verbal learning test EEG = electroencephalography; VEP = visual evoked potential; ASSR = auditory steady state response

after the first trial, subjects were asked to recall the words. Immediately thereafter, subjects underwent a memory recognition test, consisting of 15 presented words and 15 "distractors." The day-night task, a simplified version of the Stroop test suitable for children, was included in the study to assess memory and controlled processing of subjects [16].

\section{Eye Tracking}

Recording of eye movements was performed in a quiet room with dimmed illumination. Analysis was conducted with a microcomputer-based system for sampling of eye movements. Disposable electrodes (Ambu Blue Sensor N) were applied on the forehead and beside the lateral canthi of both eyes. Skin resistance was minimalized before measurements. Head movements were restrained using a fixed head support. Subjects were asked to focus on a moving dot displayed on a computer screen. Saccadic eye movements were recorded for stimulus amplitudes of approximately $15^{\circ}$ to either side. Fifteen saccades were recorded with inter-stimulus intervals varying randomly between 3 and $6 \mathrm{~s}$. Average values of saccadic peak velocity (degrees/s) of correct saccades were recorded. At least five detected saccades were necessary to include for statistical analysis. For smooth pursuit eye movements, the target moves sinusoidally at frequencies ranging from 0.3 to $1.1 \mathrm{~Hz}$. Four cycles were recorded for each stimulus frequency. The time during which the eyes were in smooth pursuit of the target was calculated and expressed as a percentage of stimulus duration [17].

\section{Executive Functioning Assessments}

The adaptive tracking test is a pursuit-tracking task and was performed as described by Borland and Nicholson [18] using customized equipment and software. The subjects were instructed to keep a dot inside a moving circle by operating a joystick. The speed of the moving circle adapted in response to subject performance. After a run-in period of $30 \mathrm{~s}$, the average tracking performance (\%) of 3.5 min was used for statistical analysis. The finger tapping test was adapted from the Halstead Reitan Test Battery [19]. Speed of finger tapping was measured for the index finger for the dominant hand; a session contained five performances of $10 \mathrm{~s}$. Subjects were instructed to tap a button as quickly as possible with the index finger of the dominant hand. The mean tapping rate was used for statistical analysis. Body sway was conducted by all subjects and assessed using a pot string meter (Celesco) based on a Wright ataxiameter, with a string attached to the waist. [20] All body movements over 2 min were integrated and expressed as sway in $\mathrm{mm}$. Before starting a measurement, subjects were asked to stand still and comfortable with their hands in a relaxed position. Subjects wore an eye cap to block sight.

\section{Electrophysiological Assessments}

Complete technical details of measurements and analysis of electrophysiological assessments are listed in Supplementary Text 1 . To measure general CNS activity, resting-state EEG with open and blocked eyes was recorded. Spectral analysis of 
the $\alpha 1, \alpha 2, \beta 1, \beta 2, \beta 3, \delta$, and $\theta$ waves was performed to calculate the power of the respective wavebands at $\mathrm{FzCz}$, $\mathrm{PzO} 1$, and $\mathrm{PzO} 2$. VEPs (visual evoked potentials) were recorded over the scalp overlying the occipital cortex. During the VEP assessment, a pattern reversal paradigm was used with two phase-changing checkerboards ( 1.0 and $0.25^{\circ}$ pattern). The oddball paradigm is a neuropsychological test to evoke event-related potentials (ERPs). During the passive oddball task, subjects were seated with EEG cap and headphones on and instructed to sit still and relax. Subjects were watching a silent movie while being presented auditory tones as frequent stimuli and infrequent stimuli. For the active oddball task, subjects were to pay attention to the tones and press a button when they heard an infrequent tone. The auditory steady-state response (ASSR) is an electrophysiological response to periodic auditory stimulation [21], thought to be generated through entrainment of neuronal populations to periodic stimuli, and reveal the integrity of neuronal networks. During the test, auditory stimuli with a $500 \mathrm{~ms}$ burst of $1 \mathrm{~ms}$ monophasic rectangular pulses were presented through headphones. The interstimulus interval was $700 \mathrm{~ms}$. The ASSR was assessed through spectral modulations and inter-trial phase coherence (ITPC).

\section{trial@home}

During the 6 days between measurements, subjects wore a Steel HR watch (Withings, Issy-les-Moulineaux, France), which is incorporated in the CHDR MORE® trial@home platform and which registered step count, heart rate, and several accelerometer-derived sleep parameters. After the final study assessments, parents and children completed a questionnaire regarding the general study experience.

\section{Statistics}

Considering the exploratory nature of this study, no formal power calculation was performed. Statistical analysis was performed with SAS v9.4 (SAS Institute, Cary, NC, USA). The difference between patients and controls was calculated via a repeated-measure mixed-model analysis of variance with fixed factors group, measurement, and group by measurement and subject as random factor. Based on the model, a minimal detectable effect size (MDES) was calculated for a hypothetical crossover study with 16 ARID1B subjects. The MDES was expressed as the proportion of the difference between ARID1B-related ID patients and controls in order to determine whether the effect size is a reasonable goal for future interventional studies. Spearman correlations between mean test outcomes and IQ and ABC subscales were calculated for tests for which a significant difference between patients and controls was demonstrated. Promasys ${ }^{\circledR} 7.3$ (Omnicomm, Fort Lauderdale, Texas, USA) was used for data management.

\section{Criteria for Candidate Endpoints}

Tests where considered fit-for-purpose when fulfilling the following requirements: (1) Tolerable, meaning subjects showed no signs of resistance during the test; (2) Conducted correctly by the study population, with more than $75 \%$ of the outcomes suitable for analysis; (3) Stable over time, defined as a coefficient of variability (CV) within the ARID1B group not higher than 50\%; (4) Statistically significant difference between healthy and control subjects; (5) Show an MDES which is less than $50 \%$ of the difference between ARID1B subjects and control subjects. Ideally, there is an association between test outcome and IQ or relevant ABC subscales.

\section{Results}

\section{Baseline Characteristics}

A total of 20 parents of patients with ARID1B-related ID were approached, of which 12 consented to participate with their child. Twelve healthy age-matched controls were included. Baseline characteristics are displayed in Table 2. Subjects with ARID1B-related ID scored highest on the hyperactivity, lethargy, and irritability $\mathrm{ABC}$ subscales. Parents found the length of study days to be too long (55\%), but none indicated they would not participate in a similar study again. The youngest ARID1B subject was 2 years old and found performing tests too difficult, after which the second study day was canceled. There were no adverse events during the conduct of this study. Individual subject characteristics are listed in Supplementary Table S1.

\section{Candidate Endpoints}

All conducted tests were assessed according to the specified criteria. Summarized results are displayed in Table 3. Individual test performance is displayed in Supplementary Table S2.

\section{Cognition}

The animal fluency test was successfully conducted by $80 \%$ of participants. One nonverbal patient did not complete the test. There was a significant difference between the two study groups and a positive correlation between the number of named animals and IQ (Fig. 1). Day-night test results did not differ between the two study groups $(p=0.133)$. The VVLT was considered too difficult and resulted in stress for the first three patients, after which the test was removed from the study. 
Table 2 Baseline characteristics

$\operatorname{ARID1B}(n=12)$

Controls $(n=12)$

$\operatorname{Age}^{1}$ (mean (range))

$\begin{array}{ll}12.6[2-31] & 11.8[2-27] \\ 9(75) & 12(100) \\ 3(17) & 1(8) \\ 74 \pm 21 & - \\ 8(67) & 12(100) \\ 8(67) & 12(100) \\ 7(58) & 0(0) \\ 12(100) & 0(0) \\ 7(58) & 0(0)\end{array}$

Sex, female $(n(\%))$

Conc. medication $(n(\%))$

$\mathrm{IQ}(\text { mean } \pm \mathrm{SD})^{2}$

Can read age appropriately $(n(\%))^{3}$

Can write age appropriately $(n(\%))^{3}$

Behavioral problems $(n(\%))^{2}$

Speech delay or impairment $(n(\%))^{2}$

$7(58)$

Vision problems $(n(\%))^{2}$
ABC subscale score $($ mean $\pm \mathrm{SD})$

Irritability

$8.3 \pm 7.4$

Lethargy

$11.2 \pm 17.2$

Stereotypic behavior

$2.4 \pm 2.1$

Hyperactivity

$13.1 \pm 10.0$

Inappropriate speech

$1.0 \pm 1.5$

${ }^{1}$ The mean age difference between patients and corresponding controls was 0.75 years. ${ }^{2}$ Data obtained from patient charts, when available. ${ }^{3}$ Parentreported

\section{Executive Functioning and Eye Tracking}

All executive functioning tests (adaptive tracking, body sway, finger tapping) were tolerable and conducted correctly.
Notably, finger tapping was the favorite assessment for $85 \%$ of subjects and $73 \%$ of parents. There was a clear and significant difference between subjects and controls for the three tests, while a correlation was present between the $\mathrm{ABC}$

Table 3 Systematic evaluation of assessments to determine suitability as endpoint in clinical trials

\begin{tabular}{|c|c|c|c|c|c|c|c|c|c|}
\hline & \multirow{2}{*}{ Test } & \multirow{2}{*}{ Tolerable $^{1}$} & \multirow{2}{*}{$\begin{array}{l}\text { Conducted } \\
\text { correctly }^{1}\end{array}$} & \multirow{2}{*}{$\begin{array}{l}\text { Repeatable } \\
\left.\text { (CV } \mathrm{CV}^{2}\right)\end{array}$} & \multicolumn{3}{|c|}{ Group difference } & \multirow{2}{*}{$\begin{array}{l}\text { MDES } \\
\text { crossover } \\
\text { design }^{3}\end{array}$} & \multirow{2}{*}{ Fit-for-purpose } \\
\hline & & & & & $\begin{array}{l}\text { ARID1B } \\
\text { Mean }\end{array}$ & $\begin{array}{c}\text { Control } \\
\text { Mean }\end{array}$ & p-value & & \\
\hline \multirow{8}{*}{ NeuroCart $^{\circledast}$} & Animal Fluency test (n) & Yes & $80 \%$ & $40 \%$ & 7.8 & 21.8 & 0.001 & $24 \%$ & Yes \\
\hline & VVLT & Yes & $33 \%^{4}$ & & & & & & No \\
\hline & Day-Night test (n) & Yes & $92 \%$ & $12 \%$ & 11.9 & 15.3 & 0.13 & & No \\
\hline & Smooth pursuit (\%) & Yes & $100 \%$ & $34 \%$ & 14.7 & 35.9 & $<0.001$ & $25 \%$ & Yes \\
\hline & $\begin{array}{l}\text { Saccadic eye movements } \\
\quad \text { Saccadic peak velocity (degr/s) } \\
\text { Saccadic reaction time (ms) }\end{array}$ & Yes & $40 \%$ & $\begin{array}{c}3 \% \\
10 \%\end{array}$ & $\begin{array}{l}515 \\
0.28\end{array}$ & $\begin{array}{l}498 \\
0.25\end{array}$ & $\begin{array}{l}0.64 \\
0.31\end{array}$ & & No \\
\hline & Adaptive tracking (\%) & Yes & $93 \%$ & $51 \%$ & 2.47 & 18.99 & $<0.001$ & $8 \%$ & Yes \\
\hline & Finger tapping (n) & Yes & $100 \%$ & $6 \%$ & 35.31 & 46.25 & 0.008 & $21 \%$ & Yes \\
\hline & Body sway (mm) & Yes & $88 \%$ & $22 \%$ & 1188 & 300 & 0.002 & $34 \%$ & Yes \\
\hline \multirow{5}{*}{ Electrophysiology } & Resting EEG & Varying & $83 \%$ & \multicolumn{5}{|c|}{ Supplementary Table S1 } & No \\
\hline & Passive oddball (MMN latency) & Varying & $87 \%$ & $22-25 \%^{5}$ & \multicolumn{3}{|c|}{ Figure 2A-C; Supplementary Table S1 } & $112 \%^{6}$ & No \\
\hline & Active oddball & Varying & $0 \%$ & & & & & & No \\
\hline & VEP & Varying & $69 \%$ & $11 \%-63 \%^{7}$ & \multicolumn{3}{|c|}{ Figure 2D; Supplementary Table S1 } & $32-73 \%^{6}$ & No \\
\hline & ASSR (ITPC) & Varying & $74 \%$ & $232 \%$ & 0.135 & 0.186 & 0.19 & & No \\
\hline \multirow{3}{*}{ trial@home } & Physical activity (step count/day) & Yes & $100 \%$ & $30 \%$ & 5559 & 7184 & 0.06 & $108 \%$ & \multirow{3}{*}{$\begin{array}{c}\text { More research } \\
\text { needed }\end{array}$} \\
\hline & $\begin{array}{l}\text { Steel HR - Sleep } \\
\text { Sleep duration (h) } \\
\text { Light sleep (\%) } \\
\text { Times to wake up (n) }\end{array}$ & Yes & $100 \%$ & $\begin{array}{l}14 \% \\
12 \% \\
51 \%\end{array}$ & $\begin{array}{c}8.7 \\
58 \% \\
3.7\end{array}$ & $\begin{array}{c}8.9 \\
51 \% \\
1.7\end{array}$ & $\begin{array}{l}0.55 \\
0.076 \\
0.006\end{array}$ & $\begin{array}{c}- \\
108 \% \\
101 \%\end{array}$ & \\
\hline & $\begin{array}{l}\text { Heart Rate } \\
\text { Daily (bpm) } \\
\text { Nocturnal (bpm) }\end{array}$ & Yes & $100 \%$ & $\begin{array}{c}- \\
5 \%\end{array}$ & $\begin{array}{l}91 \\
80\end{array}$ & $\begin{array}{l}86 \\
73\end{array}$ & $\begin{array}{c}<0.001 \\
0.009\end{array}$ & $\begin{array}{c}- \\
55 \%\end{array}$ & \\
\hline
\end{tabular}

Colors: green: suitable; red: unsuitable; yellow: indeterminate. $\mathrm{CV}=$ coefficient of variability, MDES = minimal detectable effect size, VVLT = visual verbal learning test, $\mathrm{EEG}=$ electroencephalography, $\mathrm{MMN}=$ mismatch negativity, $\mathrm{VEP}=$ visual evoked potential, ASSR $=$ auditory steady state response, ITPC $=$ inter-trial phase coherence, $\mathrm{bpm}=$ beats per minute

${ }^{1}$ By ARID1B-related ID subjects, investigator's assessment after exit interview and end-of-study questionnaire with parents; ${ }^{2}$ coefficient of variability within the group of ARID1B-related ID subjects; ${ }^{3}$ minimal detectable effect size, expressed as the proportion of the difference between patients and controls that can be detected as improvement in a crossover study with $n=16 ;{ }^{4}$ Only 1 of the first 3 ARID1B-related ID subjects was able to obtain a valid score, after which the test was removed from the study protocol; ${ }^{5}$ range of $\mathrm{CV}$ of the MMN latency at $\mathrm{Cz}$ and $\mathrm{Fz}$; ${ }^{6}$ range of MDES calculated only for parameters with a significant difference between ARID1B subjects and controls; ${ }^{7}$ range of CVs of collected parameters 
Fig. 1 Estimated group means and exploratory correlations of NeuroCart ${ }^{\circledR}$ tests. (A) Mean outcome of the animal fluency test per subject group and measurement number. (B) Linear correlation between historic IQ score and mean animal fluency test score. (C) Mean adaptive tracking test outcome per subject group and measurement number. (D) Linear correlation between the $\mathrm{ABC}$ hyperactivity subscale and mean adaptive tracking test score. (E) Mean smooth pursuit eye movement test outcome per subject group and measurement number. (F) Linear correlation between the $\mathrm{ABC}$ hyperactivity subscale and mean smooth pursuit eye movement test score. The dotted lines demarcate the two study days a Estimated mean $(95 \% \mathrm{Cl})$ Animal Fluency

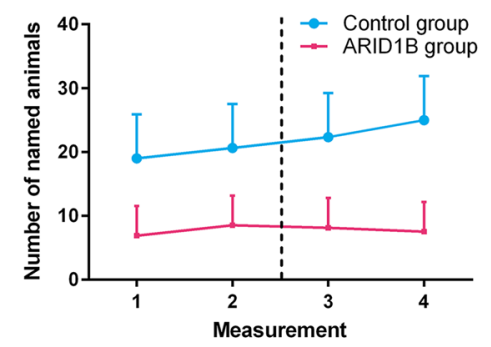

C Estimated mean $(95 \% \mathrm{Cl})$ Adaptive tracking

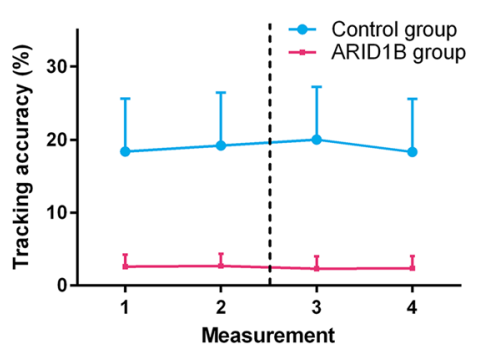

\section{e} Estimated mean (95\% Cl) Smooth pursuit

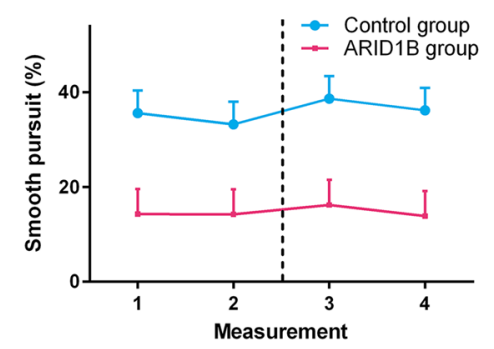

g

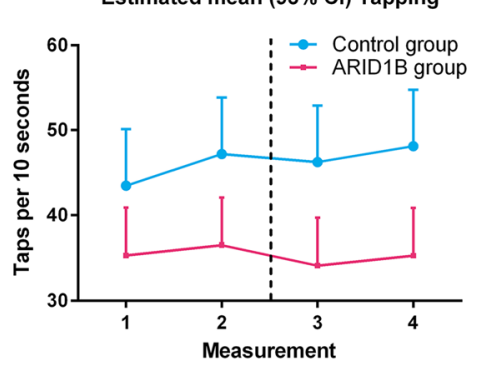

b Correlation Animal Fluency test and IQ

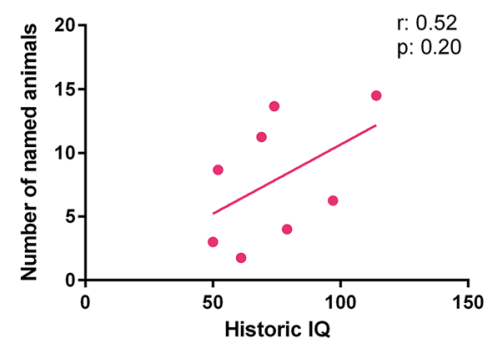

d

Correlation Adaptive tracking test and $\mathrm{ABC}$ hyperactivity

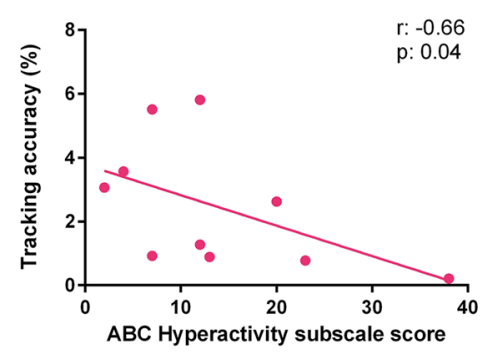

f

Correlation Smooth pursuit test and $A B C$ hyperactivity

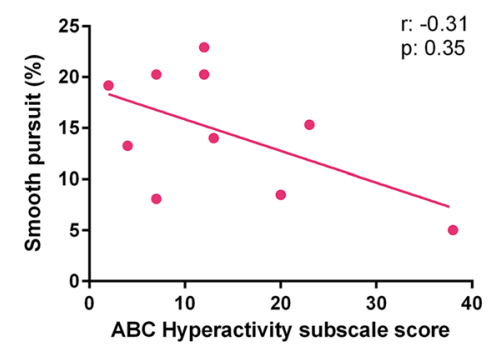

h

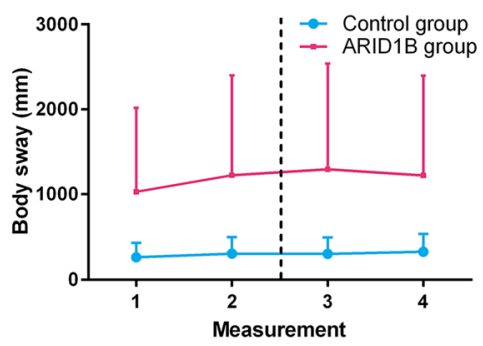

hyperactivity subscale and adaptive tracking results. Patients demonstrated a significantly lower smooth pursuit capability compared to controls and there was a correlation between mean smooth pursuit results and the $\mathrm{ABC}$ hyperactivity subscale (Fig. 1).

\section{Electrophysiological Tests}

All 24 subjects completed at least one resting-state EEG. The difference in group means of individual electrodes and EEG parameters are displayed in Supplementary Table S3. On average, a slightly higher $\alpha 2, \delta$, and $\theta$ power was detected in the occipital electrodes in ARID1B patients compared to healthy controls. The passive oddball ERP graph is displayed in Fig. 2A-C. ARID1B patients had a statistically significant difference in mismatch negativity (MMN) latency at $\mathrm{Cz}$ compared to controls (183 ms vs $141 \mathrm{~ms}, p=0.014$ ), while MMN latency at Fz and the amplitude were statistically similar. The evoked responses were different (Fig. 2A, B). The active oddball paradigm was considered too difficult for the first three patients and was subsequently removed from the study. VEP evoked response (Fig. 2D) demonstrates significantly lower amplitude and longer latency of the P100 peak, as well as a smaller N75-P100 peak-to-peak amplitude. 
a Passive oddball - Evoked response control group

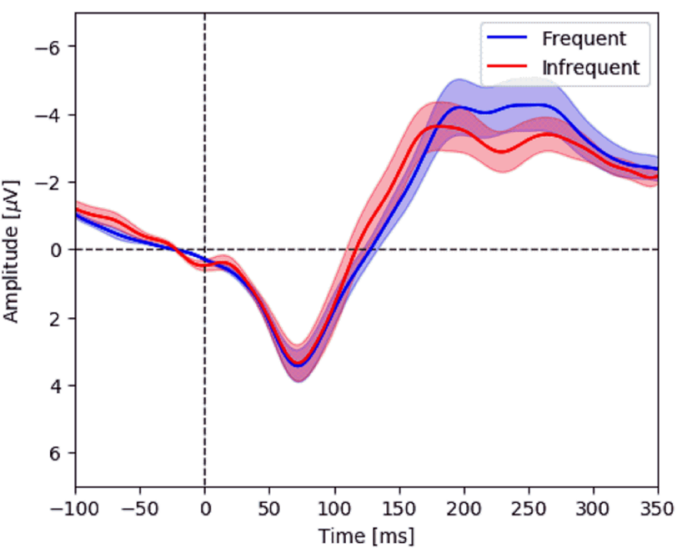

b Passive oddball - Evoked response ARID1B group

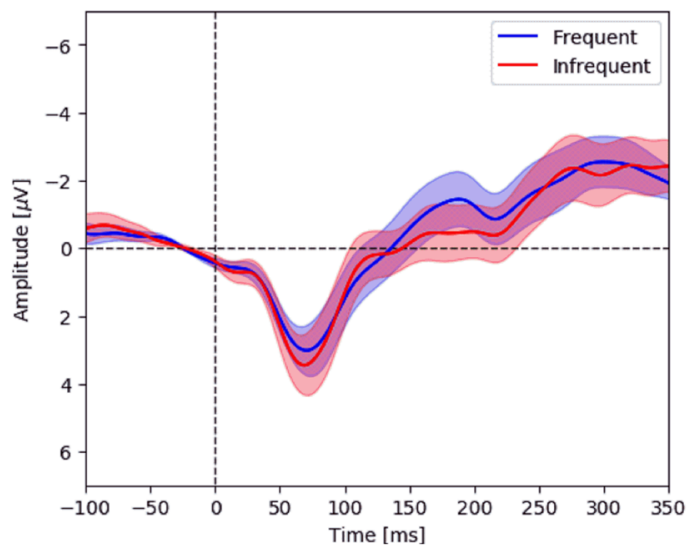

C Passive oddball - Mismatch negativity

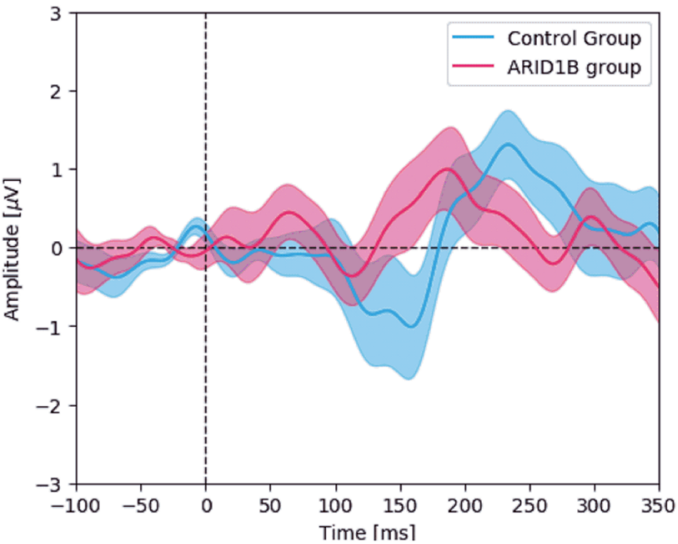

Fig. 2 ERPs of patients and controls for passive oddball and VEP assessments. (A) Grand mean of the evoked response during the passive oddball task for ARID1B subjects. (B) Grand mean of the evoked response during the passive oddball task for control group. (C) Mismatch negativity graph. (D) Visual evoked potential (VEP) ERP graph after visual stimulation with $1.0^{\circ}$ phase-changing checkerboard, including

ASSR was performed successfully in $74 \%$ of measurements, but there was no significant difference regarding ITPC and evoked power between patients and controls. Fifty percent of

\section{d Visual evoked potential - Evoked response}
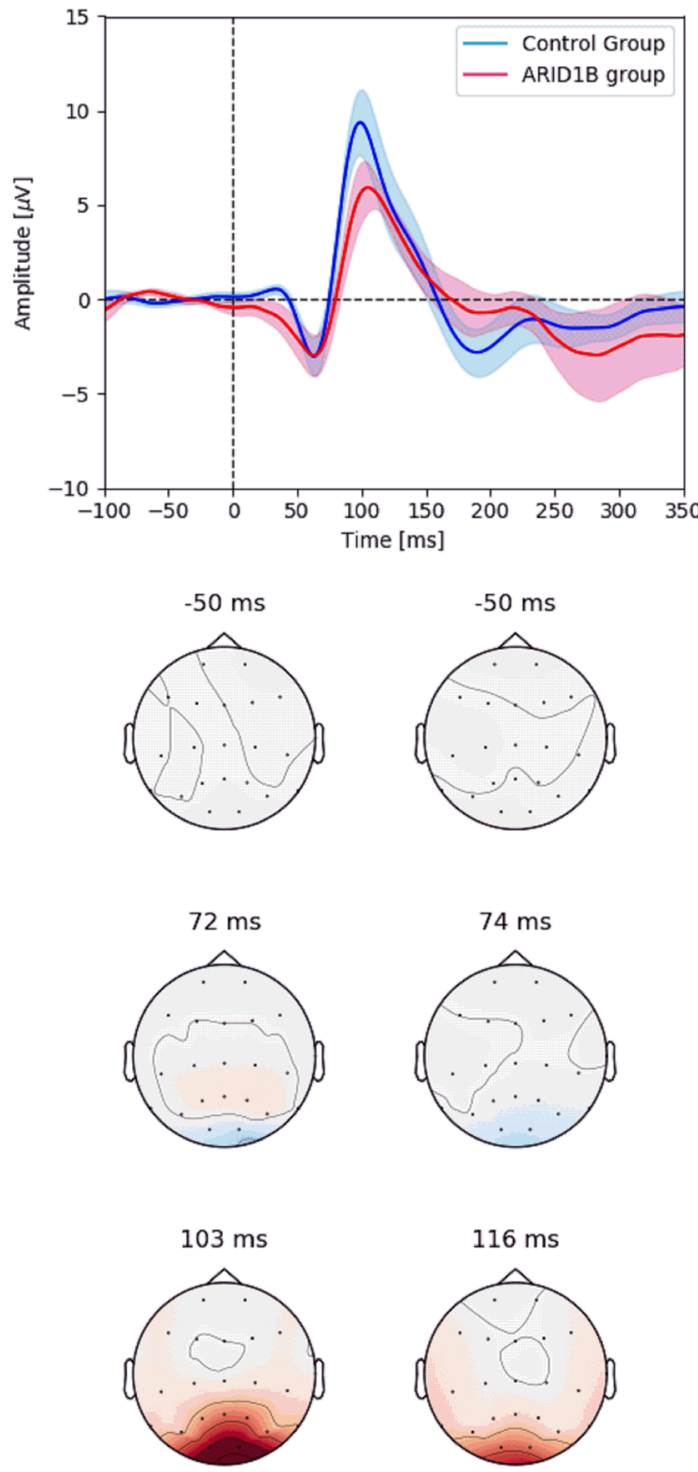

EEG heat map (left: control group; right: ARID1B group). Although subjects were also stimulated with a $0.25^{\circ}$ checkerboard, the high prevalence of refractive ametropia among ARID1B patients in combination with their disability made it impossible to determine whether all subjects saw the $0.25^{\circ}$ checkerboard clearly. A statistical summary of passive oddball and VEP analysis are listed in Supplementary Table S1

patients and controls found setting up the EEG cap to be generally uncomfortable and $42 \%$ of parents and $48 \%$ of subjects indicated EEG assessments were their least favorite. 
EEG analysis was suboptimal due to recurrent signal artifacts caused by movements of both ARID1B subjects and the younger control subjects, which led to a low overall signal quality.

\section{trial@home}

The Steel HR watch was tolerated by all subjects. $73 \%$ of subjects and $100 \%$ of parents indicated 6 days of measurements was enough or short. There was a difference in physical activity of 1625 steps per day between patients and controls, although this did not reach conventional significance $(p=$ $0.06)$. The sleep duration of both groups was similar $(8.7 \mathrm{~h}$ for patients and $8.9 \mathrm{~h}$ for controls), but patients woke up significantly more often ( 3.7 vs $1.7, p=0.006$ ). Accordingly, there was a trend towards a lower proportion of light sleep per night and a significantly higher nocturnal heart rate for patients.

\section{Discussion}

In this study, twelve patients with ARID1B-related ID and twelve age-matched controls performed a battery of noninvasive neurophysiological and neurobehavioral assessments. All assessments were reviewed for suitability as new clinical endpoints in clinical trials investigating interventions in populations with (ARID1B-related) ID. This study represents the first of its kind, providing extensive neurobehavioral and neurophysiological phenotypes of a population with an ultra-rare condition.

Of the included tests investigating cognition, the animal fluency test was identified as a promising endpoint fulfilling all predefined criteria. Considering the presence of ID in patients, the difference compared to controls was expected. However, the animal fluency test shows stability over time and the absence of a learning effect in the ARID1B group, making it suitable for the assessment of acute and mediumterm treatment effects.

Smooth pursuit eye movements fulfilled the criteria for candidate endpoints as well. In contrast, there was no statistical difference in saccadic peak velocity between patients and controls. We hypothesize this is due to the fact saccadic eye movements are a relatively preserved mechanism involving brainstem responses, while smooth pursuit is a function requiring coordination of multiple brain regions, vulnerable to developmental abnormalities [22, 23]. This has been previously demonstrated in autism and schizophrenia [24, 25]. The difference in the proportion of correctly conducted tests between smooth pursuit and saccadic eye movements was striking. We hypothesize it is more difficult to concentrate on the dot when it randomly changes position during the saccadic eye movement test, as opposed to the continuously moving dot during smooth pursuit. In the future, continuous encouragement during the test may improve the amount of analyzable results.

The three executive functioning tests were found to be suitable candidate endpoints for future studies. This is the first study to investigate these assessments in subjects with ID for this purpose, and overall test results were in line with observed symptoms. For example, patients moved considerably more than controls during the body sway test, reflecting the restlessness that ARID1B subjects exhibit. The patients' slower finger tapping may express the lethargy patients with ARID1Brelated ID suffer from. The adaptive tracking test was also positively assessed on all criteria and was correlated with the ABC hyperactivity subscale. The MDES of the ARID1B group in a crossover study with 16 subjects (1.349), relative to the found difference between the control and ARID1B group (16.52) is $8 \%$, making the adaptive tracking test the most sensitive test within this study to detect potential treatment effects.

We performed a range of electrophysiological assessments investigating general CNS activity and auditory and visual processing. Interpretation of all electrophysiological assessments was hampered due to a poor signal quality. Final analysis was performed after excluding trials of insufficient quality. For the passive oddball paradigm, ARID1B subjects appeared to exhibit a longer latency of the $\mathrm{MMN}$ at $\mathrm{Cz}$, but not the amplitude or the latency at Fz. This may reflect an impaired automatic auditory processing ability also found in one other study in subjects with ID [26]. The general evoked response appeared to be smaller for both frequent and infrequent tones (Fig. 2A, B, ), and the grand mean of the MMN (Fig. 2C) shows two small negative components before and after the average latency. This may be due to unidentified subgroups within ARID1B patients, but displaying grand means of the MMN is not ideal in this study. The MMN matures at increasing age [27]. which leads to varying MMN latencies for the different age groups. In the context of biomarker development, the estimated group means obtained via mixed model analysis may be more suitable. VEP demonstrated a longer latency and lower amplitude of the P100 peak, indicating a slower automatic visual processing ability. The complete electrophysiological substrate of these results goes beyond the scope of this paper.

Several studies have shown that pharmacological activity can alter the ERP waveform, making them an interesting, and potentially non-invasive, biomarker for drug effect in neurological disease [28]. While electrophysiological assessments can theoretically be performed by subjects of all ages, assessments were considered quite invasive for ARID1B subjects. The recurrent movement artifacts caused a significantly reduced data quality, and results should be interpreted with care. These findings show the value of our approach: EEG and ERPs could be very useful biomarkers in clinical trials, but 
recurring tests are unsuitable in this population. One could even argue that incorporation of EEG assessments in future trials would introduce bias, giving only children who are less affected by the disease the chance to repeatedly perform the assessments.

We demonstrated that an unobtrusive smartwatch can be used for home-monitoring of ID patients. Of the collected parameters, notable differences between patients and controls were found in the nocturnal parameters (number of times to wake up, nocturnal heart rate). There was a trend towards a lower physical activity level per day in patients compared to controls, although this was not a significant difference. We expect that it may be possible to detect adverse or unexpected effects of treatments, such as difficulty sleeping or apathy resulting in a decrease in physical activity using the measurements described here. However, other Withings ${ }^{\circledR}$ smartwatch models have shown a lack of reliability compared to the gold standard regarding measurement of sleep and sleep data should be interpreted with caution [29].

To summarize, we assert that the combination of animal fluency, finger tapping, body sway, adaptive tracking, smooth pursuit eye movements, and possibly homemonitoring with the Steel HR, represents a promising battery of non-invasive tests suitable for interventional studies. In our opinion, this battery of tests is non-invasive and can be conducted correctly by ID patients of 5 years and older. Furthermore, the MDES of tests calculated for a study with a feasible sample size $(n=16)$ reflect reasonable improvements of less than $50 \%$ of the difference between patients and controls.

Except for adaptive tracking, we found no statistically significant correlations with the traditional endpoints IQ and $\mathrm{ABC}$ subscales. However, significance was not expected considering this study was not powered adequately for this, and the limitations of endpoints currently used in ID trials. IQ and questionnaires have traditionally been used, but IQ has a high intra-subject variability [30], especially at a young age [31], while interpretations of questionnaires are subjective and invariably suffer from inter-rater bias [4]. Objective and standardized tests with low intra-subject variability are more suitable for early phase drug research in small patient groups. Still, improvement in parent-reported behavior certainly represents value for the individual patients and their parents. A combination of objective tests and subjective parent-reported outcomes in future trials may therefore emerge as the best paradigm.

This study has several limitations. First, the recruitment of patients focused on relatively mentally and physically competent ARID1B subjects thought to be able to tolerate traveling to the research location and being administered the test battery. Therefore, the generalizability of the study results regarding patients with severe ID is unclear. We believe the study subjects represent the population that would participate in any interventional clinical trial as well. Several cut-offs, such as for the CV and MDES, were chosen rather arbitrarily and could be more clearly defined when designing a follow-up study. We used historical IQ as a variable to correlate test outcomes with, introducing another factor of uncertainty. Historical IQ of healthy subjects was not available and could therefore not be compared between the groups. However, none of the included control subjects had learning difficulties and historical IQ was only used in the correlation with cognitive test outcomes. Although correlation of raw cognitive scores with age-adjusted standard scores such as IQ is unconventional, this cannot be avoided when no normative values of the included assessments have been determined yet. Age showed some correlation with average test outcome (Supplementary Figure S2), but this was expected and does not negatively impact the fit-for-purpose assessment of the most promising tests. A strength of this study is the repeated measurements design, generating robust data about the variability of study assessments. The included battery of tests investigated all functional CNS domains [12, 32], resulting in a comprehensive neurophysiological and neurobehavioral phenotype of ARID1B-related ID. While many psychometric properties of the candidate endpoints are unknown in the ID population, most tests have been performed in a pediatric population in the past (unpublished data) and have been extensively investigated in adult neurological disorders [12]. While the included tests have at least a theoretical relationship between disease severity and test outcome, as outlined in Table 1, this relationship must be confirmed in future studies. The included healthy controls allowed us to calculate an MDES relative to the control group, which aids in the interpretation of the effect size. Finally, we have included a relatively large cohort of patients considering the total population of patients with ARID1Brelated ID in the Netherlands.

This study shows that our approach towards the identification of fit-for-purpose endpoints in rare neurodevelopmental disorders has been successful in the case of ARID1B-related ID. During the next stage of endpoint development, the identified candidate endpoints could be included as exploratory or secondary endpoint in interventional trials in ARID1B-related ID. Furthermore, since there is a large phenotypic variability within the population, test outcomes could be compared in subgroups throughout the ARID1B spectrum. We expect our results are not specific for ARID1B-related ID. Future studies should also focus on the identified potential endpoints in patients with similar syndromes. Furthermore, prior to conducting trials investigating long-term treatment effects over a time span of years, studies aiming to uncover natural progression in test outcomes over time in patients should be performed in order to properly isolate long-term treatment effects during analysis [33]. 


\section{Conclusion}

We have identified the animal fluency test, adaptive tracking, smooth pursuit eye movement, finger tapping, and body sway as promising endpoints for clinical trials in patients with ARID1B-related ID. More research is needed in the field and physical activity and sleep monitoring. The results from this study will be used in the preparation of an interventional clinical trial investigating the effects of clonazepam in patients with ARID1B-related ID.

Acknowledgments The authors would like to thank the research assistants who conducted the tests and the data management officers and support staff at CHDR who contributed to this study. Furthermore, the authors thank Eline van der Sluijs for collection of patient data.

Required Author Forms Disclosure forms provided by the authors are available with the online version of this article.

Author Contribution MK participated in study design, conduct, and analysis and wrote the first draft of the manuscript. RZ, AC, and GJG designed the study and reviewed the manuscript; ES supervised and conducted measurements and reviewed the manuscript; MdK performed the statistical analysis and reviewed the manuscript; RJD analyzed and interpreted the EEG data and reviewed the manuscript; GS designed the study, recruited subjects, and reviewed the manuscript.

Funding information This study was funded by the Centre for Human Drug Research.

Data Availability The data that support the findings of this study are available from the corresponding author upon reasonable request.

\section{Compliance with Ethical Standards}

This study was approved by the Beoordeling Ethiek Biomedisch Onderzoek (BEBO) Foundation Review Board (Assen, the Netherlands). The study was conducted according to the Dutch Act on Medical Research Involving Human Subjects, the Dutch codes of conduct regarding medical research with minors and expression of objection by people with mental disabilities and in compliance with good clinical practice.

Conflict of Interest The authors declare that they have no conflict of interest.

Open Access This article is licensed under a Creative Commons Attribution 4.0 International License, which permits use, sharing, adaptation, distribution and reproduction in any medium or format, as long as you give appropriate credit to the original author(s) and the source, provide a link to the Creative Commons licence, and indicate if changes were made. The images or other third party material in this article are included in the article's Creative Commons licence, unless indicated otherwise in a credit line to the material. If material is not included in the article's Creative Commons licence and your intended use is not permitted by statutory regulation or exceeds the permitted use, you will need to obtain permission directly from the copyright holder. To view a copy of this licence, visit http://creativecommons.org/licenses/by/4.0/.

\section{References}

1. van Karnebeek CDM, Bowden K, Berry-Kravis E. Treatment of Neurogenetic Developmental Conditions: From 2016 into the Future [Internet]. Vol. 65, Pediatric Neurology. Elsevier Inc; 2016. p. 1-13. Available from: https://doi.org/10.1016/j. pediatrneurol.2016.07.010

2. Lee AW, Ventola P, Budimirovic D, Berry-Kravis E, Visootsak J. Clinical development of targeted fragile $\mathrm{X}$ syndrome treatments: An industry perspective. Vol. 8, Brain Sciences. 2018. p. 1-15.

3. Berry-Kravis E, Hagerman R, Visootsak J, Budimirovic D, Kaufmann WE, Cherubini M, et al. Arbaclofen in fragile X syndrome: Results of phase 3 trials. J Neurodev Disord. 2017;9(1):118.

4. Budimirovic DB, Berry-Kravis E, Erickson CA, Hall SS, Hessl D, Reiss AL, et al. Updated report on tools to measure outcomes of clinical trials in fragile X syndrome. J Neurodev Disord. 2017;9(1): $1-36$.

5. Abrahamyan L, Feldman BM, Tomlinson G, Faughnan ME, Johnson SR, Diamond IR, et al. Alternative designs for clinical trials in rare diseases. Am J Med Genet Part C Semin Med Genet. 2016;172(4):313-31.

6. Santen GWE, Aten E, Sun Y, Almomani R, Gilissen C, Nielsen M, et al. Mutations in SWI/SNF chromatin remodeling complex gene ARID1B cause Coffin-Siris syndrome. Nat Genet [Internet]. 2012;44(4):379-80. Available from: https://doi.org/10.1038/ng. 2217

7. Tsurusaki Y, Okamoto N, Ohashi H, Kosho T, Imai Y, Hibi-Ko Y, et al. Mutations affecting components of the SWI/SNF complex cause Coffin-Siris syndrome. Nat Genet [Internet]. 2012;44(4): 376-8. Available from: https://doi.org/10.1038/ng.2219

8. van der Sluijs E (P J)., Jansen S, Vergano SA, Adachi-Fukuda M, Alanay Y, AlKindy A, et al. The ARID1B spectrum in 143 patients: from nonsyndromic intellectual disability to Coffin-Siris syndrome. Genet Med. 2018;13-24.

9. Santen GWE, Clayton-Smith J, Adachi-Fukuda M, AlKindy A, Baban A, Berry K, et al. The ARID1B phenotype: What we have learned so far. Am J Med Genet Part C Semin Med Genet. 2014;166(3):276-89.

10. Jung EM, Moffat JJ, Liu J, Dravid SM, Gurumurthy CB, Kim WY. Arid1b haploinsufficiency disrupts cortical interneuron development and mouse behavior. Nat Neurosci [Internet]. 2017;20(12): 1694-707. Available from: https://doi.org/10.1038/s41593-0170013-0

11. Sahin M, Jones SR, Sweeney JA, Berry-Kravis E, Connors BW, Ewen JB, et al. Discovering translational biomarkers in neurodevelopmental disorders. Nat Rev Drug Discov [Internet]. 2018;18(april):7-8. Available from: https://doi.org/10.1038/ d41573-018-00010-7

12. Groeneveld GJ, Hay JL, Van Gerven JM. Measuring blood-brain barrier penetration using the NeuroCart, a CNS test battery [Internet]. Vol. 20, Drug Discovery Today: Technologies. The Author(s); 2016. p. 27-34. Available from: https://doi.org/10. 1016/j.ddtec.2016.07.004

13. Aman MG, Singh NN, Stewart AW, Field CJ. The aberrant behavior checklist: a behavior rating scale for the assessment of treatment effects. Am J Ment Defic [Internet]. 1985;89(5):485-91. Available from: http://www.ncbi.nlm.nih.gov/pubmed/3993694

14. Tombaugh, T. N., Kozak, J . \& Rees L. Normative data stratified by age and education for two measures of verbal fluency : FAS and Animal Naming . Archives of Clinical Neuropsychology , 14, 167177 . 1999;0(2):13-4. 
15. van der Elst W, van Boxtel MPJ, van Breukelen GJP, Jolles J. Rey's verbal learning test: Normative data for 1855 healthy participants aged 24-81 years and the influence of age, sex, education, and mode of presentation. J Int Neuropsychol Soc. 2005;11(3):290-302.

16. Gerstadt CL, Hong YJ, Diamond A. The relationship between cognition and action: Performance of 3.5-7 year olds on a Stroop-like day night test. Cognition. 1994;53(1):129-53.

17. Van Steveninck AL, Schoemaker HC, Pieters MSM, Kroon R, Breimer DD, Cohen AF. A comparison of the sensitivities of adaptive tracking, eye movement analysis, and visual analog lines to the effects of incremental doses of temazepam in healthy volunteers. Clin Pharmacol Ther. 1991;50(2):172-80.

18. Borland R, Nicholson A. Visual motor co-ordination and dynamic visual acuity. Br J Clin Pharmacol. 1984;18(1 S):69S-72S.

19. Dikmen SS, Heaton RK, Grant I, Temkin NR. Test-retest reliability and practice effects of expanded Halstead-Reitan Neuropsychological Test Battery. J Int Neuropsychol Soc [Internet]. 1999 May 1 [cited 2019 Sep 29];5(4):346-56. Available from: https://www.cambridge.org/core/product/ identifier/S1355617799544056/type/journal_article

20. van Steveninck AL, Gieschke R, Schoemaker HC, Pieters MSM, Kroon JM, Breimer DD, et al. Pharmacodynamic interactions of diazepam and intravenous alcohol at pseudo steady state. Psychopharmacology (Berl). 1993;110(4):471-8.

21. Brenner CA, Krishnan GP, Vohs JL, Ahn WY, Hetrick WP, Morzorati SL, et al. Steady state responses: Electrophysiological assessment of sensory function in schizophrenia. Schizophr Bull. 2009;35(6):1065-77.

22. Mustari MJ, Ono S, Das VE. Signal processing and distribution in cortical-brainstem pathways for smooth pursuit eye movements. Ann N Y Acad Sci. 2009;1164:147-54.

23. McDowell JE, Dyckman KA, Austin BP, Clementz BA. Neurophysiology and neuroanatomy of reflexive and volitional saccades: Evidence from studies of humans. Brain Cogn [Internet]. 2008;68(3):255-70. Available from: https://doi.org/10. 1016/j.bandc.2008.08.016

24. Wilkes BJ, Carson TB, Patel KP, Lewis MH, White KD. Oculomotor performance in children with high-functioning
Autism Spectrum Disorders. Res Dev Disabil [Internet]. 2015;38: 338-44. Available from: https://doi.org/10.1016/j.ridd.2014.12. 022

25. O'Driscoll GA, Callahan BL. Smooth pursuit in schizophrenia: A meta-analytic review of research since 1993. Brain Cogn [Internet]. 2008;68(3):359-70. Available from: https://doi.org/10.1016/j. bandc.2008.08.023

26. Knotht IS, Lippé S. Event-related potential alterations in fragile $\mathrm{X}$ syndrome. Vol. 6, Frontiers in Human Neuroscience. 2012. p. 117.

27. Shafer VL, Morr ML, Kreuzer JA, Kurtzberg D. Maturation of mismatch negativity in school-age children. Ear Hear. 2000;21(3): 242-51.

28. Blokland A, Prickaerts J, Van Duinen M, Sambeth A. The use of EEG parameters as predictors of drug effects on cognition. Eur J Pharmacol [Internet]. 2015;759:163-8. Available from: https://doi. org/10.1016/j.ejphar.2015.03.031

29. Gruwez A, Bruyneel AV, Bruyneel M. The validity of two commercially-available sleep trackers and actigraphy for assessment of sleep parameters in obstructive sleep apnea patients. PLoS One. 2019;14(1):1-11.

30. Krassowski E, Plante E. IQ variability in children with SLI: Implications for use of cognitive referencing in determining SLI. J Commun Disord. 1997;30(1):1-9.

31. Schuerger JM, Witt AC. THE TEMPORAL STABILITY OF INDIVIDUALLY TESTED INTELLIGENCE. J Clin Psychol. 1989;45(2):294-302.

32. De Visser SJ, Van Der Post JP, De Waal PP, Cornet F, Cohen AF, Van Gerven JMA. Biomarkers for the effects of benzodiazepines in healthy volunteers. Br J Clin Pharmacol. 2003;55(1):39-50.

33. Santen GWE, Cohen AF. Rare disease specialists and clinical pharmacologists unite: Increase collection of longitudinal data! Br J Clin Pharmacol. 2019;

Publisher's Note Springer Nature remains neutral with regard to jurisdictional claims in published maps and institutional affiliations. 